\title{
DLCQ Strings and Branched Covers of Torii
}

\author{
Gordon W. Semenoffa *

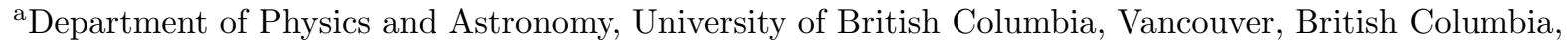 \\ Canada V6T 1 Z1.
}

Presented at the Light-Cone Workshop

"Light-cone Physics: Particles and Strings"

Trento, Italy, September, 2001

In this lecture I will review some results about the discrete light-cone quantization (DLCQ) of strings and some connections of the results with matrix string theory. I will review arguments which show that, in the path integral representation of the thermal free energy of a string, the compactifications which are necessary to obtain discrete light-cone quantization constrains the integral over all Riemann surfaces of a given genus to the set of those Riemann surfaces which are branched covers of a particular torus. I then review an explicit check of this result at genus 1 . I discuss the intriguing suggestion that these branched covers of a torus are related to those which are found in a certain limit of the matrix string model.

\section{Preamble}

In this lecture I will review some aspects of the discrete light-cone quantization (DLCQ) of strings. This is a summary of work which has appeared in a series of publications about this subject, its relationship with the Matrix model of M-theory and related issues, [1]-[5].

One of the essential points will be that, when the light-cone is compactified, the degrees of freedom of the string are thinned somewhat. We will see this by examining the thermodynamic partition function. The thinning of degrees of freedom can be quantified geometrically and summarized in a simple statement about string worldsheets: The set of string worldsheets in DLCQ is the set of those Riemann surfaces which are branched covers of a particular torus, rather than the bigger set of all Riemann surfaces. This means that the string fluctuates less. A further lesson is that these branched covers appear naturally in certain models of random matrices [6]-[12]. The example that we are particularly interested in here is matrix string theory where the limit of weak string

\footnotetext{
*This work is supported in part by NSERC of Canada.
}

coupling of the model is related to string degrees of freedom living on branched covers.

After discussing the general result, we will illustrate by doing three different computations of the same quantity: the thermodynamic partition function of discrete light-cone quantized strings. The first computation uses the path Polyakov path integral. The second computation takes the operator quantization of the free string and solves for the spectrum and then constructs the thermodynamic partition function from that spectrum. The third computation begins with a certain limit of the matrix model of M-theory. In this limit the model reduces to a statistical theory of eigenvalues of the matrices which live on branched covers of a torus. The partition function should be compared with that of free type II strings. In all three cases, we obtain an identical result.

An essential tool that we use is the thermodynamic partition function. In our case, we simply regard it as a generating function for the energy spectrum of free string theory. Of course, a certain amount of caution must always be used when discussing string theory at finite temperature. Closed string theory is a theory of quantum 
gravity. In a gravitational system, a homogeneous state with finite energy density has the Jeans instability where it collapses to form black holes. The Jeans instability sets in at long wave-lengths so one might expect that the volume of a system in which conventional thermodynamics is valid is limited so as to cut off those wave-lengths. In fact, the limiting volume is greater if the string coupling is less. We can get a crude estimate of the bound by comparing the entropy of black hole states with that of a thermal gas of gravitons.

Black holes have a large amount of entropy and can easily dominate the density of states of a quantum gravitational system. A perfect gas of gravitons has energy and entropy given roughly by

$$
E=T^{D} V
$$

and

$$
S=T^{D-1} V
$$

respectively. A black hole with energy $E$ has Schwarzchild radius $R \sim E^{1 /(D-3)}$ (in Planck units) and entropy proportional to the area of its event horizon, $S \sim(E)^{\frac{D-2}{D-3}}$. In the set of all possible states, the perfect gas states dominate over black hole states if they have more entropy,

$$
T^{D-1} V>(E)^{\frac{D-2}{D-3}} \approx\left(T^{D} V\right)^{\frac{D-2}{D-3}}
$$

which, with linear dimension given by $L=$ $V^{1 /(D-1)}$, yields the formula

$$
L<\left(\frac{L_{S}}{L_{P}}\right)^{\frac{D-2}{D-1}} \frac{1}{T^{\frac{2 D-3}{D-1}}}
$$

where we have re-scaled the temperature and length so that they are in string units, rather than Planck units. Thus, stringy states are excited if $T \sim 1$ (in fact, to the accuracy of our estimates, the Hagedorn transition happens when $T \sim 1$ ). If the string coupling, $g_{s}$ is small, then $L_{S} / L_{P}$ is greater than one. The number of strings lengths in the maximal volume is roughly $L_{S} / L_{P}$ which is large at weak string coupling.

\section{DLCQ of the string: Path integral}

In this Section we will review a result about discrete light cone quantization of strings using the path integral which were first obtained in ref.[2]. The vacuum energy of the string is computed by the Polyakov path integral

$F=-\sum_{g, \sigma} g_{s}^{2 g-2} \int\left[d h_{g} d X d \Psi\right] e^{-S[X, \Psi, h]}$

where the action is

$S=\frac{1}{4 \pi \alpha^{\prime}} \int \sqrt{h}\left(\partial_{a} X^{\mu} \partial^{a} X^{\mu}-2 \pi i \alpha^{\prime} \Psi^{\mu} \gamma \cdot \nabla \Psi^{\mu}\right)$

The string coupling constant is $g_{s}$ and its powers weight the genus, $g=0,1, \ldots$, of the string's worldsheet. There is also a sum over spin structures, $\sigma$ which, with the appropriate weights, imposes the GSO projection. For each value of the genus, $g,\left[d h_{g}\right]$ is an integration measure over all metrics of that genus and is normalized by dividing by the volume of the worldsheet reparameterization and Weyl groups.

To get finite temperature $T=1 / \beta$ we compactify the Euclidean time,

$$
\left(X^{0}, \vec{X}, X^{9}\right) \sim\left(X^{0}+\beta, \vec{X}, X^{9}\right)
$$

This also requires appropriate modification of the GSO projection to make the target space fermions anti-periodic.

DLCQ requires compactification of the lightcone in Minkowski space. We do this by making the identification of Minkowski space coordinates

$$
\frac{1}{\sqrt{2}}\left(t+x^{9}\right) \sim \frac{1}{\sqrt{2}}\left(t+x^{9}\right)+2 \pi R
$$

In Euclidean space this is the complex identification

$$
\left(X^{0}, \vec{X}, X^{9}\right) \sim\left(X^{0}, \vec{X}, X^{9}\right)+\sqrt{2} \pi R(i, 0,1)(4)
$$

With this compactification the GSO projection is unmodified.

In order to implement compactifications, we must include topological sectors in the path integral. In these sectors, the string worldsheet

\footnotetext{
${ }^{2}$ Here, for concreteness, we use the Neveu-SchwarzRamond superstring. It is important to keep in mind that our considerations which lead to the result of this Section would apply equally well to the bosonic sector of any string theory, including the bosonic string. The relationship with matrix theory which we shall discuss later, the most appropriate theory is the Green-Schwarz superstring.
} 
wraps the compact directions. To study the general case, we begin by developing some notation. The worldsheet is a Riemann surface $\Sigma_{g}$ of genus $g$ whose homology group $H_{1}\left(\Sigma_{g}\right)$ is generated by the a complete set of cycles (non-contractable closed curves) which can always be separated into two sets and chosen to have the canonical intersection property

$$
\begin{aligned}
& a_{1}, a_{2}, \ldots, a_{g}, b_{1}, b_{2}, \ldots, b_{g} \\
& a_{i} \cap a_{j}=\emptyset, b_{i} \cap b_{j}=\emptyset, a_{i} \cap b_{j}=\delta_{i j}
\end{aligned}
$$

In addition, there is a basis of holomorphic differentials $\omega_{i} \in H^{1}\left(\Sigma_{g}\right)$ (1-forms obeying $\left.d \omega^{i}=0\right)$ with the properties

$$
\oint_{a_{i}} \omega_{j}=\delta_{i j} \quad, \quad \oint_{b_{i}} \omega_{j}=\Omega_{i j}
$$

where $\Omega=\Omega_{1}+i \Omega_{2}$ is the period matrix. It is complex, symmetric and has positive definite imaginary part.

When the worldsheet wraps a compact dimension, the bosonic coordinates of the string $X^{\mu}$ should have a multi-valued part which changes by $\beta \cdot$ integer $\cdot(1,0,0)$ or $\sqrt{2} \pi R$.integer $\cdot(i, 0,1)$ as it is moved along a homology cycle. To deal with single-valued quantities, we consider the 1 -forms $d X^{\mu}$ which can be expanded in holomorphic and anti-holomorphic differentials and exact parts,

$$
\begin{array}{r}
d X^{0}=\sum_{i=1}^{g}\left(\lambda_{i} \omega_{i}+\bar{\lambda}_{i} \bar{\omega}_{i}\right)+\text { exact } \\
d \vec{X}=\text { exact } \\
d X^{9}=\sum_{i=1}^{g}\left(\gamma_{i} \omega_{i}+\bar{\gamma}_{i} \bar{\omega}_{i}\right)+\text { exact }
\end{array}
$$

The holomorphic and anti-holomorphic 1-forms account for the holonomy. Indeed, we require that

$$
\begin{aligned}
& \oint_{a_{i}} d X^{0}=\beta n_{i}+\sqrt{2} \pi R i p_{i} \\
& \oint_{b_{i}} d X^{0}=\beta m_{i}+\sqrt{2} \pi R i q_{i} \\
& \oint_{a_{i}} d X^{9}=\sqrt{2} \pi R p_{i} \quad, \quad \oint_{b_{i}} d X^{9}=\sqrt{2} \pi R q_{i}
\end{aligned}
$$

With eqn.(6), we use these equations to solve for the constants in eqn.(7). Then, with the formula

$$
\begin{array}{r}
\int \omega_{i} \bar{\omega}_{j}=\sum_{k=1}^{g}\left(\oint_{a_{k}} \omega_{i} \oint_{b_{k}} \bar{\omega}_{j}-\oint_{b_{k}} \omega_{i} \oint_{a_{k}} \bar{\omega}_{j}\right)= \\
=-2 i\left(\Omega_{2}\right)_{i j}
\end{array}
$$

we can find the part of the string action which contains the winding integers,

$$
\begin{array}{r}
S=\frac{\beta^{2}}{4 \pi \alpha^{\prime}}\left(n \Omega^{\dagger}-m\right) \Omega_{2}^{-1}(\Omega n-m)+ \\
+2 \pi i \frac{\sqrt{2} \beta R}{4 \pi \alpha^{\prime}} \frac{1}{2}\left[\left(p \Omega^{\dagger}-q\right) \Omega_{2}^{-1}(\Omega n-m)\right. \\
\left.\quad+\left(n \Omega^{\dagger}-m\right) \Omega_{2}^{-1}(\Omega p-q)\right]+\ldots
\end{array}
$$

The integers $p_{i}$ and $q_{i}$ appear linearly in a purely imaginary term in the action. Furthermore, since they come from the compactification of the light cone, this is the only place that they will appear in the entire string path integral (unlike $n_{i}$ and $m_{i}$ which, because of the modification of the GSO projection at finite temperature, should appear in the weights of the sum over spin structures).

The action must be exponentiated and summed over $p_{i}$ and $q_{i}$. There are two cases to consider. First there is the special case where all $m_{i}=0=n_{i}$. This gives the zero temperature limit of the free energy, that is, the vacuum energy times the space-time volume. In this case, entire topological part of the action vanishes and, after dividing by the space-time volume, the vacuum energy density does not depend on the null compactification radius, $R$. The summation over $p_{i}$ and $q_{i}$ give a divergent overall factor.

The fact that the vacuum energy density does not depend on $R$ can be understood from the idea of Seiberg and Sen [13]-[14] together with T-duality. The space-time with a compact null dimension can be obtained by beginning with a space-time with a compact spatial dimension, then doing an infinite boost along that direction and simultaneously shrinking the size of the original space-like compactification radius to zero. However, because of T-duality, in closed string theory, the sum of zero point energies of the strings at zero compactification radius is just identical to that sum at infinite compactification 
radius. The partition function computes energies, not energy densities. Thus, the vacuum energy density must be unaffected by DLCQ and the infinite factor which arises is just the ratio of the radii of the infinite and zero sized circles. This can easily be seen by explicitly working through the exercise of using a boosted spatial compactification

$$
X^{+} \sim X^{+}+\sqrt{2} \pi R^{1} \Lambda, X^{-} \sim X^{-}-\sqrt{2} \pi R^{1} / \Lambda
$$

where $\Lambda$ is the boost parameter and seeing that in the limit of infinite boost $\Lambda \rightarrow \infty$ and vanishing spatial radius $R^{1} \rightarrow 0$ such that the product $R^{1} \Lambda=\sqrt{2} R$ is held constant, the vacuum energy divided by $R_{1}$ (and other $R^{1}$-independent factors) to get energy density becomes $R$ independent.

In the following we will consider the situation where the zero temperature limit of the free energy, the vacuum energy, has been subtracted. Then we can assume that at least some of the integers $n_{i}, m_{i}$ are non-zero. Then, the result of summing the exponential of the action over $p_{i}, q_{i}$ will be periodic Dirac delta functions. It can be shown that these delta functions impose a linear constraint on the period matrix of the worldsheet. The net effect is to insert into the path integral measure the following expression,

$$
\begin{array}{r}
\sum_{m n r s} e^{-\frac{\beta^{2}}{4 \pi \alpha^{\prime}}\left(n \Omega^{\dagger}-m\right) \Omega_{2}^{-1}(\Omega n-m)} \nu^{-2 g}\left|\operatorname{det} \Omega_{2}\right| . \\
\cdot \prod_{j=1}^{g} \delta\left(\sum_{i=1}^{g}\left(n_{i}+\frac{i}{\nu} r_{i}\right) \Omega_{i j}-\left(m_{j}+\frac{i}{\nu} s_{j}\right)\right) \\
\cdot U(m, n, \sigma)
\end{array}
$$

where $\nu=\sqrt{2} \beta R / 4 \pi \alpha^{\prime}$ is a fixed constant and $U(m, n, \sigma)$ makes the appropriate modification of the GSO projection. This modification is discussed with explicit examples for genus 1 in ref. [15], for example. The only other place that any information about the compactification enters the theory is in the integration over the zero modes in the bosonic part of the path integral. This produces a factor of the total volume of the spacetime which should be divided out of both sides of eqn.(1) to obtain the thermodynamic free energy density.
This is our central result, that the net effect of compactifications is to simply insert the expression eqn.(17) into the path integral measure. This result was first obtained in ref. [2]. Since the first homology group of the 2-sphere is trivial, the first order in perturbation theory which is affected is at genus 1. A similar constraint on the modular parameters at genus 1 was found for scattering amplitudes and argued to also exist and resemble eqn.(12) at higher genera in ref.[16]-[17]. It would be interesting to generalize our further observations about the geometric interpretation of this constraint (in Section 5) to scattering amplitudes.

\section{Partition function of the Bosonic string at genus 1}

It is interesting to see what the result of the previous Section tells us about the DLCQ finite temperature partition function of the Bosonic string at genus 1. The usual torus amplitude of the bosonic string is

$$
\frac{F}{V}=-\int_{\mathcal{F}} \frac{d \tau_{1} d \tau_{2}}{\tau_{2}}\left(\frac{1}{4 \pi \alpha^{\prime} \tau_{2}}\right)^{13}|\eta(\tau)|^{-48}
$$

where the complex modular parameter $\tau=\tau_{1}+$ $i \tau_{2}$ is integrated over the fundamental domain of the torus,

$\mathcal{F} \equiv\left\{\tau|| \tau_{1}\left|\leq \frac{1}{2} ;\right| \tau \mid \geq 1 ; \tau_{2}>0\right\}$

Our observations in the previous Section tell us that we obtain the finite temperature DLCQ partition function by inserting the expression in eqn.(12) with $g=1$ into the integrand (and set $\mathrm{U}=1$ ). When $g=1$ that expression takes the form

$\sum_{m n r s} \frac{\tau_{2}}{\nu^{2}} e^{-\frac{\beta^{2}|\tau m-n|^{2}}{4 \pi \alpha^{\prime} \tau_{2}}} \delta\left(\left(n+\frac{i}{\nu} r\right) \tau-\left(\left(m+\frac{i}{\nu} s\right)\right)\right.$

The insertion results in

$\frac{F}{V}=-\sum_{\tau \in \mathcal{F}} \frac{\nu^{-2} e^{-\frac{\beta^{2}|n \tau-m|^{2}}{4 \pi \alpha^{\prime} \tau_{2}}}}{n^{2}+\nu^{-2} r^{2}}\left(\frac{1}{4 \pi \alpha^{\prime} \tau_{2}}\right)^{13}|\eta(\tau)|^{-48}(14)$

where

$\tau=\frac{m+\frac{i}{\nu} s}{n+\frac{i}{\nu} r}$ 
In eqn.(14) we have integrated the delta function (with the necessary Jacobian). Here, we see that the moduli space of the torus is completely discretized. We will find a geometric interpretation of this discretization in Section 5. In the next Section we pause to see whether this result is consistent with what one would obtain by an alternative technique - working out the spectrum of the free DLCQ string by operator methods and constructing the thermodynamic partition function by tracing the appropriate density matrix.

\section{Operator derivation of the partition function for bosonic string at genus 1}

In this Section, in order to check the validity of eqn.(14), we will compute the genus 1 contribution to the finite temperature partition function using operator quantization in the (discrete) light-cone gauge. For simplicity, we will use the bosonic string as an example. This derivation as well as one for the type II superstring which will be discussed in the following Section was given in more detail in [1].

In light-cone quantization the mass spectrum of the Bosonic string on 26-dimensional Minkowski space is given by the eigenvalues of the mass operator

$M^{2}=2 P^{+} P^{-}-\vec{P}^{2}=\frac{2}{\alpha^{\prime}}\left(L_{0}+\tilde{L}_{0}-2\right)$

which also satisfy the level matching condition

$$
L_{0}-\tilde{L}_{0}=0
$$

where

$$
L_{0}=\frac{1}{2} \sum_{n-1}^{\infty} \alpha_{-n} \cdot \alpha_{n} \quad, \quad \tilde{L}_{0}=\frac{1}{2} \sum_{n=1}^{\infty} \tilde{\alpha}_{-n} \cdot \tilde{\alpha}_{n}
$$

and

$$
\left[\alpha_{m}^{i}, \alpha_{n}^{j}\right]=m \delta_{m+n, 0} \delta^{i j},\left[\tilde{\alpha}_{m}^{i}, \tilde{\alpha}_{n}^{j}\right]=m \delta_{m+n, 0} \delta^{i j}
$$

with $i, j=1, \ldots, 24$ are the usual string oscillators. The light-cone momenta are

$P^{ \pm}=\frac{1}{\sqrt{2}}\left(P^{0} \pm P^{D-1}\right) \quad, \vec{P}=\left(P^{1}, \ldots, P^{D-2}\right)$
When we compactify the null direction to get DLCQ of the string we must make the coordinate identification

$$
X^{+} \sim X^{+}+2 \pi R
$$

This modifies the spectrum of the string in two ways. First, it quantizes the momentum conjugate to $X^{+},\left(\right.$since $\left.\left[X^{+}, P^{-}\right]=i\right)$,

$$
P^{-}=N / R
$$

Second, it introduces a wrapping number $w$ of the string world sheet on the compact dimension. It turns out that this wrapping number does not appear in the mass shell condition, eqn.(16), which remains unmodified. The only modification is to the level matching condition which becomes

$L_{0}-\tilde{L}_{0}=N w$

The usual light-cone quantization is recovered if we let $R \rightarrow \infty$ and $N \rightarrow \infty$ holding $P^{-}$fixed. Then the spectrum of $P^{-}$reverts to a continuum and states with $w \neq 0$ go to infinite mass.

Here we will consider the thermodynamic partition function of the free string. We will begin by deriving it using the spectrum which we discussed above. The spectrum of the free string is identical to the spectrum of a tower of free particles. The free energy of a second quantized free bosonic particle of mass $M$ is given by

$F=\frac{1}{\beta} \operatorname{Tr} \ln \left(1-e^{-\beta P^{0}}\right)$

where we have subtracted the temperature independent vacuum energy. Our first step is to Taylor expand this expression to produce

$F=-\sum_{k=1}^{\infty} \frac{1}{k \beta} \operatorname{Tr} e^{-k \beta P^{0}}$

Here, we see that the many particle free energy is expanded in terms of heat kernels which propagate some positive number of intervals $\beta$ in imaginary time.

The energy in terms of light-cone momenta is

$P^{0}=\frac{\left(P^{+}+P^{-}\right)}{\sqrt{2}}=\frac{1}{\sqrt{2}}\left(\frac{N}{R}+\frac{\vec{P}^{2}+M^{2}}{2 N / R}\right)$ 
We see from this expression that, if the energy is to be positive, then $N$ must also be positive. Furthermore, there is no finite energy physical state with $N=0$.

The free energy density is

$$
\frac{F}{V}=-\int \frac{d \vec{p}}{(2 \pi)^{D-2}} \sum_{k, N=1}^{\infty} \frac{e^{-\frac{\beta k N}{\sqrt{2} R}-\frac{\vec{p}^{2}+M^{2}}{2 \sqrt{2} N / k \beta R}}}{\sqrt{2} \pi \beta R k}
$$

We have divided both sides by the spatial volume which is $V=\sqrt{2} \pi R$ (transverse volume). We have taken a trace over the momenta. The spectrum of $\mathrm{P}^{+}$is discrete and tracing over it leads to the sum over $N$. The integral over transverse momenta $\vec{p}$ with measure (transverse volume) $\iint d^{D-2} p(2 \pi)^{D-2}$ is the trace over the spectrum of $\vec{P}$. Finally, integrating over $\vec{p}$, we get

$\frac{F}{V}=-\sum_{k, N=1}^{\infty}\left(\frac{N}{\sqrt{2} \pi k \beta R}\right)^{D / 2} \frac{e^{-\frac{\beta N k}{\sqrt{2} R}-\frac{\beta R k}{2 \sqrt{2} N} M^{2}}}{N}$

We can derive the string partition function from this expression by summing it over the masses of physical particle states which occur in the free string spectrum. In order to do this, we use the mass shell condition, eqn.(16), to write the mass-dependent part of eqn.(21) as an operator and take its trace over physical states of the string. This means evaluating the trace over states which obey the level matching condition eqn.(17) of the operator

$$
\exp \left(-2 \pi \tau_{2}\left(L_{0}+\tilde{L}_{0}-2\right)\right)
$$

where $\tau_{2}=\frac{k}{N} \frac{\sqrt{2} \beta R}{4 \pi \alpha^{\prime}}=\frac{k}{N} \nu$ with $\nu$ the quantity which was defined after eqn.eqn.(12).

We project onto that part of the spectrum which obeys the level matching condition using a fourier integral,

$$
\delta\left(L_{0}-\tilde{L}_{0}-w N\right)=\int_{0}^{1} d \tau_{1} e^{2 \pi i \tau_{1}\left(L_{0}-\tilde{L}_{0}-N w\right)}
$$

so that the sum over physical masses is equivalent to computing

$$
\int_{0}^{1} d \tau_{1}\left|\operatorname{Tr} e^{2 \pi i \tau L_{0}}\right|^{2} e^{-2 \pi i \tau_{1} w N+4 \pi \tau_{2}}
$$

This expression is the only place where the wrapping number $w$ will appear and it should be summed over all integers. When summed, it gives a periodic delta function,

$$
\sum_{w=-\infty}^{\infty} \exp \left(-2 \pi i \tau_{1} w N\right)=\frac{1}{N} \sum_{r=0}^{N-1} \delta\left(\tau_{1}-r / N\right)
$$

In this way, the sum over physical masses takes the form

$\frac{1}{N} \sum_{r=0}^{N-1}\left|\operatorname{Tr} \exp \left(2 \pi i \tau L_{0}\right)\right|_{\tau_{1}=r / N}^{2} e^{4 \pi \tau_{2}}$

with

$\tau=\tau_{1}+i \tau_{2}=\frac{r+i k \nu}{N}$

The trace over the spectrum of $L_{0}$ is given in terms of the Dedekind eta-function

$$
\operatorname{Tr} e^{2 \pi i \tau L_{0}}=\prod_{n}\left(\frac{1}{1-e^{2 \pi i \tau n}}\right)^{24} \equiv e^{2 \pi i \tau} \eta^{-24}(\tau)
$$

The final result for the thermodynamic partition function of the Bosonic string at temperature $1 / \beta$ with light-cone radius $R$ is

$$
\begin{array}{r}
\frac{F}{V}=-\sum_{k, N=1}^{\infty} \sum_{r=0}^{N-1} \frac{1}{N^{2}}\left(\frac{1}{4 \pi^{2} \alpha^{\prime} \tau_{2}}\right)^{13} . \\
\cdot e^{-\frac{k^{2} \beta^{2}}{4 \pi \alpha^{\prime} \tau_{2}}}|\eta(\tau)|^{-48}
\end{array}
$$

(We have put $\mathrm{D}=26$.) This expression is not yet identical to eqn.(14). It is summed over three integers which specify the modular parameter in eqn.(25) instead of the four integers which specify the modular parameter in eqn.(15) and the two parameters have a different domain. Indeed, they can be shown to be equal using the "Polchinski trick" [18]. The essential observation is that the summand

$$
\left(\frac{1}{4 \pi \alpha^{\prime} \tau_{2}}\right)^{13}|\eta(\tau)|^{-48}
$$

is invariant under modular transformations

$$
\tau \rightarrow \frac{a+b \tau}{c+d \tau} \quad a b c d \in \mathcal{Z} \quad a d-b c=1
$$


We can always find a modular transformation which sets the integer $n$ in eqn.(15) and eqn.(14) to zero. This modular transformation also maps the point $\tau=\frac{m+\frac{i}{\nu} s}{n+\frac{i}{\nu} r}$ in the fundamental domain to a point $\tau^{\prime}=\frac{m^{\prime}+\frac{i}{\nu} s^{\prime}}{\frac{i}{\nu} r^{\prime}}$ in the strip $\tau_{2} \in[0,1] \tau_{1}>$ 0 . The result is that the sum over the three integers $k N r$ in the domain $\tau_{2} \in[0,1] \tau_{1}>0$ is equivalent to the sum over four integers mnrs over the domain $\mathcal{F}$. With this observation, eqn.(26) and eqn.(14) are seen to be identical.

\section{Type II superstring at genus 1}

As another concrete example, consider the type II superstring torus amplitude

$$
\begin{array}{r}
\frac{F}{V}=-\int_{\mathcal{F}} \frac{d^{2} \tau}{4 \tau_{2}} \frac{1}{\left(4 \pi^{2} \alpha^{\prime} \tau_{2}\right)^{5}} \frac{1}{|\eta(\tau)|^{24}} . \\
\cdot\left[\theta_{2}^{4} \bar{\theta}_{2}^{4}+\theta_{3}^{4} \bar{\theta}_{3}^{4}+\theta_{4}^{4} \bar{\theta}_{4}^{4}+\left(\theta_{2}^{4} \bar{\theta}_{4}^{4}+\theta_{4}^{4} \bar{\theta}_{2}^{4}\right)\right. \\
\left.\quad-\left(\theta_{2}^{4} \bar{\theta}_{3}^{4}+\theta_{3}^{4} \bar{\theta}_{2}^{4}\right)-\left(\theta_{3}^{4} \bar{\theta}_{4}^{4}+\theta_{4}^{4} \bar{\theta}_{3}^{4}\right)\right]
\end{array}
$$

where $\theta_{k}(0, \tau)$ are Jacobi theta functions and $\eta(\tau)$ is the Dedekind eta-function. The modification of this formula by the null compactification can be found using eqn.(12),

$$
\begin{aligned}
& \frac{F}{V}=-\sum_{\tau \in \mathcal{F}} \frac{\nu^{-2}}{n^{2}+\nu^{-2} r^{2}} \frac{e^{-\frac{\beta^{2}|n \tau-m|^{2}}{4 \pi \alpha^{\prime} \tau_{2}}}}{\left(4 \pi^{2} \alpha^{\prime} \tau_{2}\right)^{5}} \frac{1}{4|\eta(\tau)|^{24}} . \\
& {\left[\theta_{2}^{4} \bar{\theta}_{2}^{4}+\theta_{3}^{4} \bar{\theta}_{3}^{4}+\theta_{4}^{4} \bar{\theta}_{4}^{4}+(-1)^{m+n}\left(\theta_{2}^{4} \bar{\theta}_{4}^{4}+\theta_{4}^{4} \bar{\theta}_{2}^{4}\right)\right.} \\
& \left.-(-1)^{n}\left(\theta_{2}^{4} \bar{\theta}_{3}^{4}+\theta_{3}^{4} \bar{\theta}_{2}^{4}\right)-(-1)^{m}\left(\theta_{3}^{4} \bar{\theta}_{4}^{4}+\theta_{4}^{4} \bar{\theta}_{3}^{4}\right)\right]
\end{aligned}
$$

where $\tau=\left(m+\frac{i}{\nu} s\right) /\left(n+\frac{i}{\nu} r\right)$ and one should sum over the integers so that $\tau$ is in the fundamental domain, $\mathcal{F}$. It was shown in [1] that this is precisely the finite temperature partition function that is obtained for the type II superstring by operator methods and DLCQ.

Part of the argument showed that it is also equal to the partition function of the GreenSchwarz formulation of the type II superstring. The equivalence is demonstrated by using modular transformations and identities for theta functions. Another presentation of the same function, which we shall have use for later is to rewrite eqn.(28) as the Hecke operator [19] acting on the partition function of a superconformal field the- ory, with torus worldsheet and target space $R^{8}$ :

$$
\begin{aligned}
\frac{F}{V}= & -\frac{1}{\sqrt{2} \pi R \beta} \mathcal{H}\left[e^{-\beta / \sqrt{2} R}\right] * \\
& {\left[\left(\frac{1}{4 \pi^{2} \alpha^{\prime} \tau_{2}}\right)^{4} \frac{1}{|\eta(\tau)|^{24}}\left|\theta_{2}(0, \tau)\right|^{8}\right]_{\tau=i \nu}(29) }
\end{aligned}
$$

The factor in front is the ratio of volumes of $R^{8}$ and $R^{9} \times S^{1}$ with compactified light cone. The action of $\mathcal{H}[p]$ on a function $\phi(\tau, \bar{\tau})$ is defined by

$\mathcal{H}[p] * \phi(\tau, \bar{\tau})=\sum_{N=0}^{\infty} \frac{p^{N}}{N} \sum_{\substack{k r=N \\ s \bmod k}} \phi\left(\frac{r \tau+s}{k}, \frac{r \bar{\tau}+s}{k}\right)$

where the sum is over $k$ and $r$ which are divisors of $N$ with the restriction that $r$ is an odd integer. This operation appears in the quantization of superconformal field theories on symmetric orbifolds 20]-22]. We will construct an explicit version of it in our discussion of matrix strings in a later Section.

\subsection{Geometrical interpretation of the con- straint on Riemann surfaces}

In the sectors with non-zero wrappings, the delta function in eqn.(12) restricts the integration over metrics in the string path integral to those for which the period matrix obeys the constraint

$\sum_{i=1}^{g}\left(n_{i}+\frac{i}{\nu} r_{i}\right) \Omega_{i j}-\left(m_{j}+\frac{i}{\nu} s_{j}\right)=0$

for all combinations of the $4 g$ integers $m_{i}, n_{i}, r_{i}, s_{i}$ such that $\Omega$ is in a fundamental domain. This is clearly a subset of all Riemann surfaces. It is interesting to ask whether one can characterize this subset in a convenient way. In this Section we will review a theorem about the classification which was proved in [2].

A hint about this classification can be found by counting the number of moduli of the Riemann surface that eqn. 311 removes. The number of complex moduli of a Riemann surface $\Sigma_{g}$ is $3 g-3$ when $g>1$. Since the columns of the period matrix are linearly independent vectors, eqn.(31) contains $g$ independent complex constraints. Thus the complex dimension $3 g-3$ 
is reduced to $2 g-3$ and there is further discrete data contained in the integers. 3

$2 g-3$ is the dimension of the moduli space of a genus $g$ branched cover of a torus. Indeed, given $n$ branch points, the genus is one plus the number of branch cuts, $g=1+n / 2$. The moduli are the independent positions of the branch points, $n-$ 1 where one is subtracted for overall translation invariance. The resulting dimension is $2 g-3$, identical to the one which we found above.

In fact, we can prove the following Theorem: $\Sigma_{g}$ is a branched cover of a 2 -torus $T^{2}$ with modular parameter $\frac{i}{\nu}$ if and only if $\Omega_{i j}$ obeys eqn.(31), for some choice of integers $m_{i}, n_{i}, r_{i}$ and $s_{i}$.

Proof: The generators of the first homology group of $T^{2}$ are two closed loops $(\alpha, \beta)$ which span the vector space $H_{1}\left(T^{2}, \mathcal{C}\right)$. The dual vector space, the first cohomology group, $H^{1}\left(T^{2}, \mathcal{C}\right)$ is spanned by the basis of holomorphic and anti-holomorphic differentials $\gamma$ and $\bar{\gamma}$. They can be normalized as,

$$
\begin{array}{ll}
\oint_{\alpha} \gamma=1, & \oint_{\beta} \gamma=\frac{i}{\nu} \\
\oint_{\alpha} \bar{\gamma}=1, & \oint_{\beta} \bar{\gamma}=-\frac{i}{\nu}
\end{array}
$$

The Riemann surface $\Sigma_{g}$ is a branched cover of $T^{2}$ if there exists a continuous holomorphic map $f$, such that

$\Sigma_{g} \stackrel{f}{\longrightarrow} T^{2}$

The map $f$ takes closed loops on $\Sigma_{g}$ to closed loops on $T^{2}$. In particular, the generators eqn.(5) must map as

$\left(a_{i}, b_{j}\right) \stackrel{f}{\longrightarrow}\left(n_{i} \alpha+r_{i} \beta, m_{j} \alpha+s_{j} \beta\right)$

for some integers $m_{i}, n_{i}, r_{i}, s_{i}$. This gives a mapping between the vector spaces $H_{1}\left(\Sigma_{g}, \mathcal{C}\right)$ and $H_{1}\left(T^{2}, \mathcal{C}\right)$. The pull-back of this mapping on the dual vector spaces

$H^{1}\left(T^{2}, \mathcal{C}\right) \stackrel{f^{*}}{\longrightarrow} H^{1}\left(\Sigma_{g}, \mathcal{C}\right)$

${ }^{3}$ This could alternatively be viewed as a partial discretization of the moduli space. One would expect that, when the compactifications are removed, either $\beta \rightarrow \infty$ or $R \rightarrow \infty$, the discrete data assembles itself to a "continuum limit" which restores the $g$ complex dimensions of moduli space. is defined by its action on the basis,

$$
\begin{array}{r}
a_{i} \circ f^{*}(\gamma)=\oint_{a_{i}} f^{*}(\gamma) \equiv f\left(a_{i}\right) \circ \gamma= \\
=n_{i} \oint_{\alpha} \gamma+r_{i} \oint_{\beta} \gamma=n_{i}+\frac{i}{\nu} r_{i} \\
b_{j} \circ f^{*}(\gamma)=\oint_{b_{j}} f^{*}(\gamma) \equiv f\left(b_{j}\right) \circ \gamma= \\
=m_{j} \oint_{\alpha} \gamma+s_{j} \oint_{\beta} \gamma=m_{j}+\frac{i}{\nu} s_{j}
\end{array}
$$

Consider the $g$ particular elements of $H_{1}\left(\Sigma_{g}, \mathcal{C}\right)$, which we shall call $c_{i}$. They are linear combination of the cycles which are given by

$c_{j}=\sum_{i=1}^{g} a_{i} \Omega_{i j}-b_{j}$

It can be checked from the definition of the period matrix eqn.(5) (and the fact that it is symmetric) that the operation of any holomorphic differential, $\eta$, annihilates all of these combinations of cycles,

$c_{j} \circ \eta=\sum_{i=1}^{g} \Omega_{i j} \oint_{a_{i}} \eta-\oint_{b_{j}} \eta=0$

The holomorphic nature of the mapping, $f$, guarantees that $f^{*}(\gamma)$ is a holomorphic differential on $\Sigma_{g}$. The equation eqn.(37) should therefore also apply to $f^{*}$. Then it follows that

$$
\begin{gathered}
0=c_{j} \circ f^{*}(\gamma)=f\left(c_{j}\right) \circ \gamma= \\
=\sum_{i=1}^{g}\left(n_{i}+\frac{i}{\nu} r_{i}\right) \Omega_{i j}-\left(m_{j}+\frac{i}{\nu} s_{j}\right)
\end{gathered}
$$

which is the constraint on the period matrix in eq.(31). Thus, we have proven that, if $\Sigma_{g}$ is a branched cover, then its period matrix obeys eqn.(31). We have further found an interpretation of the integers, they are those which appear in the projections of the homology generators of $\Sigma_{g}$ onto the homology generators of $T^{2}$ which are induced by the covering map.

To prove the converse, we must now show that if the constraint in eqn.(31) is satisfied, then a covering map, $f$, exists. We will demonstrate this 
by explicit construction. Consider the line integral of a linear combination of the holomorphic differentials on $\Sigma_{g}$,

$z(P)=\int_{P_{0}}^{P} \sum_{k=1}^{g} \lambda_{k} \omega_{k}$

with $P_{0}$ a fixed base-point. We want to view this integral as a mapping between points $P \in \Sigma_{g}$ and the numbers $z(P)$ which are points in the complex plane. Of course, this mapping depends on the path of integration which is chosen and is multi-valued. In fact, the best we can do is to adjust the mapping so that $z(P)$ is a function from $\Sigma_{g}$ to the quotient of the complex plane by a lattice.

For this, we wish to choose the coefficients $\lambda_{k}$ such that this integral defines a map from points $P \in \Sigma_{g}$ to the torus $z(P) \in T^{2}$ whose holomorphic coordinates are the complex numbers $z(P)$ with the identification

$$
z \sim z+p+\frac{i}{\nu} q
$$

where $p$ and $q$ are integers. The integral depends on the path of integration. If the path is changed by a combination of homology cycles of $\Sigma_{g}$, say $k_{i} a_{i}+l_{i} b_{i}$ where $k_{i}, l_{i}$ are integers, the integral on the right-hand side of eqn.(39) changes by

$$
\delta z=\sum_{j} \lambda_{j}\left(k_{j}+\sum_{i} \Omega_{j i} l_{i}\right)
$$

$\lambda_{k}$ must be chosen so that this change is integer multiples of the periods of $T^{2}$. This can easily be done if $\Omega$ obeys eqn.(31). Then, with the choice

$$
\lambda_{i}=n_{i}+\frac{i}{\nu} r_{i}
$$

we see that

$$
\begin{gathered}
\delta z=\sum_{j}\left(n_{j}+\frac{i}{\nu} r_{j}\right) k_{j}+\sum_{i}\left(m_{i}+\frac{i}{\nu} s_{i}\right) l_{i}= \\
=\text { integer }+\frac{i}{\nu} \cdot \text { integer }
\end{gathered}
$$

and we have constructed an explicit covering map, $z(P)$. Q.E.D.

\section{The thermodynamic partition function of matrix string theory}

In this Section, we will review the argument of ref.11 which computes the thermodynamic partition function of the free string limit of matrix string theory.

Matrix string theory is a 1+1-dimensional maximally supersymmetric Yang-Mills theory on a spatial circle. Its Hamiltonian is (The original derivation is in ref. [23]. For details, see the reviews in ref.24] 25 the arguments in ref.[1].)

$$
\begin{array}{r}
H=\frac{R}{2} \int_{0}^{1} d \sigma_{1} \operatorname{Tr}\left[\Pi_{i}^{2}+\frac{1}{4 \pi^{2} \alpha^{\prime 2}}\left(D_{1} X^{i}\right)^{2}-\right. \\
-\frac{1}{4 \pi^{2} \alpha^{\prime 3} g_{s}^{2}}\left[X^{i}, X^{j}\right]^{2}+\frac{E^{2}}{g_{s}^{2} \alpha^{\prime}} \\
\left.-\frac{1}{2 \pi \alpha^{\prime 3 / 2} g_{s}} \psi^{T} \gamma_{i}\left[X^{i}, \psi\right]-\frac{i}{2 \pi \alpha^{\prime}} \psi^{T} \gamma \cdot D_{1} \psi\right]
\end{array}
$$

Here, $\Pi^{i}$ is the canonical momentum conjugate to $X^{i}, E$ is the electric field and canonical conjugate to the gauge field $A_{\mu}$ which appears in the covariant derivative $(\partial-i[A$,$) the gauge group$ is $\mathrm{SU}(\mathrm{N})$ and all degrees of freedom transform in the adjoint representation. Here, the coordinates have been rescaled so that $\sigma_{1} \in[0,1)$ and all variables are periodic under the spatial translation, $X^{i}\left(\tau, \sigma_{1}\right)=X^{i}\left(\tau, \sigma_{1}+1\right)$. This Hamiltonian should be augmented by a Gauss' law constraint which requires that all physical states are gauge invariant.

This theory is conjectured to describe M-theory with two compactification, a spatial dimension with radius $g_{s} \sqrt{\alpha^{\prime}}$ which, when the radius is small gives type IIA superstring theory and a null dimension with radius R to give DLCQ [26,27,23]. We should identify the light-cone momenta of matrix string theory as $P^{-}=N / R$ and $P^{+}=H$ and the quantum states are the gauge invariant eigenstates of $H$. The thermodynamic partition function is

$$
\begin{array}{r}
Z=e^{-\beta F}=\operatorname{Tr} e^{-\beta P^{0}}=\operatorname{Tr} e^{-\beta\left(P^{+}+P^{-}\right) / \sqrt{2}} \\
=\sum_{N=0}^{\infty} e^{-N \beta / \sqrt{2} R^{+}} \operatorname{Tr}\left\{e^{-\beta H / \sqrt{2}}\right\}
\end{array}
$$

The trace of $\exp (-\beta H / \sqrt{2})$ over gauge invariant states has the standard path integral expression 
of a finite temperature Yang-Mills theory partition function

$Z[\beta]=\sum_{N=0}^{\infty} e^{-\frac{\beta N}{\sqrt{2} R}} \int[d A d X d \psi] e^{-S_{E}\left[A, X^{i}, \psi\right]}$

where $S_{E}$ is the Euclidean action. The time variable in the Yang-Mills theory is Euclidean and compact with domain $\sigma_{2} \in[0, \beta]$. By re-scaling the Euclidean coordinates so that they live in the domain $\sigma_{a} \in[0,1)$ we can write the action as

$$
\begin{array}{r}
S_{E}=\frac{1}{4 \pi \alpha^{\prime}} \int_{g^{2} d^{2} \sigma \sqrt{|g|} \operatorname{Tr}\left\{g^{a b} D_{a} X^{i} D_{b} X^{i}+\right.}+\frac{1}{|g|} F_{12}^{2}-\frac{1}{\alpha^{\prime} g_{s}^{2}} \sum_{i<j}\left[X^{i}, X^{j}\right]^{2}- \\
\left.-i \psi^{T} \gamma \cdot D \psi-\frac{1}{\sqrt{\alpha^{\prime}} g_{s}} \psi^{T} \gamma^{i}\left[X^{i}, \psi\right]\right\}
\end{array}
$$

We have re-scaled Euclidean time $\sigma_{2}$ so that the integration is over a box of area one, $0 \leq \sigma_{a}<1$, and $\beta$ appears as a factor in various coupling constants which have been absorbed into the metric. $\left(\gamma^{\mu}, \gamma^{i}\right)$ are the ten-dimensional Gamma matrices in the Majorana-Weyl representation. Bosonic variables are periodic in both space and time and fermionic variables are periodic in space and anti-periodic in time. The anti-periodicity of the fermion field in the Euclidean time comes from taking the trace in eqn.(41). Thus, the space that the Yang-Mills theory is defined on is a torus. The metric of the torus is [1]:

$g_{a b}=\left(\begin{array}{cc}1 & 0 \\ 0 & \nu^{2}\end{array}\right)$

where $\nu=\frac{\sqrt{2} \beta R}{4 \pi \alpha^{\prime}}$ is a familiar parameter. This is just the metric of the underlying torus which we found in the previous Sections. Furthermore, the Yang-Mills coupling constant is given by $g_{Y M}^{2}=$ $1 / \alpha^{\prime} g_{s}$.

The limit $g_{s} \rightarrow 0$ of this theory was developed by Dijkgraaf, Verlinde and Verlinde 23|. The hypothesis is that the degrees of freedom which become perturbative strings in the limit $g_{s} \rightarrow 0$ are the simultaneous eigenvalues of the matrices which must commute if they are to have finite energy. Commuting matrices can be simultaneously diagonalized by a gauge transformation

$X^{i}(\sigma)=U(\sigma) X_{D}^{i}(\sigma) U^{-1}(\sigma)$

$$
\begin{aligned}
\psi(\sigma) & =U(\sigma) \psi_{D}(\sigma) U^{-1}(\sigma) \\
A_{\mu}(\sigma) & =i U(\sigma)\left(\partial_{\mu}-i A_{\mu}^{D}(\sigma)\right) U^{-1}(\sigma)
\end{aligned}
$$

where $X_{D}^{i}, \psi_{D}$ and $A_{\mu}^{D}$ are diagonal. The fields $X^{i}, \psi$ and $A_{\mu}$ live on the torus with metric given in eqn.(44). Eigenvalues of these matrices solve polynomial equations with doubly periodic coefficients. They are generally not single-valued functions on the torus. Instead, they are single-valued functions on branched covers of the torus. Since they are all diagonalized by the same unitary transformation, they must all live on the same branched cover.

In this way we see that the degrees of freedom which correspond to perturbative strings live on branched covers of a basic torus, precisely those branched covers which, by the arguments of Section 2 and 5, are the worldsheets of DLCQ strings. It would be very interesting to show that an asymptotic expansion of the matrix string free energy in eqn.(41) reproduces the string perturbation theory in eqn.(11) with the constraint eqn.(12) inserted. Although there is a large amount of literature in this direction [29]-33], this has yet to be demonstrated in detail for genera greater than one. At genus 1, it is indeed the case, as was shown in ref. [1]. We will review the essential features of the argument here.

To get genus 1, we simply take the diagonal matrices to live on a genus 1 (unbranched) cover of the torus. The unbranched cover arises when we assume that the diagonal matrices in eqn.(45) are smooth functions, without branch points. Then they have boundary conditions which are periodid f up to permutations. For example, for the scalar fields

$\left(X_{D}^{i}\right)_{\alpha}\left(\sigma_{1}+1, \sigma_{2}\right)=\left(X_{D}^{i}\right)_{P(\alpha)}\left(\sigma_{1}, \sigma_{2}\right)$
$\left(X_{D}^{i}\right)_{\alpha}\left(\sigma_{1}, \sigma_{2}+1\right)=\left(X_{D}^{i}\right)_{Q(\alpha)}\left(\sigma_{1}, \sigma_{2}\right)$

where $\alpha=1, \ldots, N, P(\alpha)$ and $Q(\alpha)$ are permutations. The same permutation acts on all of the fields since they are all diagonalized by the same unitary matrix in eqn.(45).

Consistency requires that the two permutations commute,

$P Q=Q P$

${ }^{4}$ Keep in mind that Fermions are anti-periodic in the Euclidean time direction. 
The partition function is now given by

$$
Z=\sum_{N=0}^{\infty} \frac{1}{N !} e^{-N \beta / \sqrt{2} R} \sum_{\substack{P, Q \\ P Q=Q P}} Z(P, Q),
$$

where

$$
Z(P, Q)=\int[d A d X d \psi] e^{-S_{\mathrm{diag}}}
$$

with the boundary conditions (46) determined by $P$ and $Q$. In eqn.(48), for each $N$, we have divided by the volume of the Weyl group, $N$ !, which reflects the fact that the eigenvalues are defined up to a global permutation 5 . The action is

$$
\begin{aligned}
S_{\text {diag }}= & \frac{1}{4 \pi \alpha^{\prime}} \int d^{2} \sigma \sqrt{|g|} \sum_{\alpha a=1}^{N}\left\{g^{a b} \partial_{a} X_{\alpha}^{i} \partial_{b} X_{\alpha}^{i}+\right. \\
& \left.+\frac{g_{s}^{2} \alpha^{\prime}}{|g|}\left(\partial_{1} A_{2 \alpha}-\partial_{2} A_{1 \alpha}\right)^{2}-i \psi_{\alpha}^{T} \gamma \cdot \nabla \psi_{\alpha}\right\}
\end{aligned}
$$

and with the boundary conditions (46).

It is useful at this point to review some of the salient points about commuting permutations, for which we follow the appendix of ref. [7]. Another useful resource is ref. 34.

Consider a fixed permutation $P$ of a set of $N$ elements. It can be decomposed into cycles which are subsets of the $N$ elements such that the permutation interchanges elements cyclically inside each subset. For example, for $N=9$, the permutation which can be denoted by

$$
\alpha=\left(\begin{array}{lllllllll}
1 & 2 & 3 & 4 & 5 & 6 & 7 & 8 & 9 \\
6 & 4 & 1 & 2 & 5 & 3 & 8 & 9 & 7
\end{array}\right)
$$

can be decomposed into the product of disjoint cycles

$$
\alpha=(163)(24)(5)(789)
$$

\footnotetext{
${ }^{5}$ From the M-theory point of view, this factor gives Boltzmann statistics to the D0-branes 28 The possibility of introducing different weights in the sum over pairs of commuting permutations has been discussed in [7]. We shall show in what follows that the correct parfition function for the type IIA string emerges from eqn.(48) only when all of the pairs $(P, Q)$ have the same weight. It turns out that, at genus 1, the gauge field integral in eqn.(48) is trivial and we will drop it from now on [1]. There is a beautiful suggestion in refs. 33,35 that at higher genera it gives the string coupling constant correct factor $g_{s}^{2 g-2}$.
}

Consider such a decomposition of $P$. Suppose that the number of cycles of length $k$ is $r_{k}$, so that $N=\sum_{k} k r_{k}$. Clearly, the minimum value of $k$ is one and the maximum is $N . r_{k}$ are nonnegative integers. (In the above example, $r_{1}=$ $1, r_{2}=1, r_{3}=2$.) Denote the elements of the set $\{1,2, \ldots, N\}$ by the three index notation based on how they transform under $P$ :

$a_{n}^{k, \gamma}(k=1, \ldots, N) ;\left(\gamma=1, \ldots, r_{k}\right):(n=1, \ldots, k)(51)$

This element of $\{1, \ldots, N\}$ is the nth element of the $\gamma^{\prime}$ th cycle of length $k$. The action of the permutation on this element is then

$P\left(a_{n}^{k, \gamma}\right)=a_{n+1 \bmod k}^{k, \gamma}$

Let $Q$ be a permutation which commutes with $P$. Consider its action on the cycle

$$
\left(a_{1}^{k, \gamma}, \ldots, a_{k}^{k, \gamma}\right)
$$

of P. Eq. (52) implies that

$Q\left(P\left(a_{n}^{k, \gamma}\right)\right)=Q\left(a_{n+1 \bmod k}^{k, \gamma}\right)$

Since $P Q=Q P$, this equation can be written as

$P\left(Q\left(a_{n}^{k, \gamma}\right)\right)=Q\left(a_{n+1 \bmod k}^{k, \gamma}\right)$

This means that

$$
\left(Q\left(a_{1}^{k, \gamma}\right),, \ldots, Q\left(a_{k}^{k, \gamma}\right)\right)
$$

is a cycle of length $k$ in $P$, i.e. that

$$
\begin{gathered}
\left(Q\left(a_{1}^{k, \gamma}\right),, \ldots, Q\left(a_{k}^{k, \gamma}\right)\right)= \\
=\left(a_{s(k, \gamma)}^{k, \pi_{k}(\gamma)}, \ldots, a_{s(k, \gamma)+k-1 \bmod k}^{k, \pi_{k}(\gamma)}\right)
\end{gathered}
$$

Hence, there exists a permutation $\pi_{k}(\gamma)$ of the $r_{k}$ elements of the set of cycles of length $k$ which is induced by $Q$. Also, besides permuting the cycles of $P$ of equal length, $Q$ can also do a cyclic permutation of each cycle by the integer $s(k, \gamma)$ which can take the values $1, . ., k$. Thus, the only allowed action of $Q$ is

$Q\left(a_{n}^{k, \gamma}\right)=a_{n+s(k, \gamma) \bmod k}^{k, \pi_{k}(\gamma)}$ 
Consider a set of diagonal components of fields obeying the boundary conditions

$X_{\alpha}^{i}\left(\sigma_{1}+1, \sigma_{2}\right)=X_{P(\alpha)}^{i}\left(\sigma_{1}, \sigma_{2}\right)$,

$X_{\alpha}^{i}\left(\sigma_{1}, \sigma_{2}+1\right)=X_{Q(\alpha)}^{i}\left(\sigma_{1}, \sigma_{2}\right)$.

Consider those which occur in the cycles of length $k$ of $P$ and re-label them according to elements of the cycle,

$$
\begin{gathered}
X_{a_{n}^{k \gamma}}^{i}\left(\sigma_{1}+1, \sigma_{2}\right)=X_{a_{n+1}^{k \gamma}}^{i}\left(\sigma_{1}, \sigma_{2}\right), \\
X_{a^{k \gamma n}}^{i}\left(\sigma_{1}, \sigma_{2}+1\right)=X_{a_{n+s}^{i k(\gamma)}}^{i}\left(\sigma_{1}, \sigma_{2}\right) .
\end{gathered}
$$

For each fixed $\gamma$, we fuse these fields together into a single function which has the property

$$
\begin{gathered}
X_{a^{k \gamma}}^{i}\left(\sigma_{1}+k, \sigma_{2}\right)=X_{a^{k \gamma}}^{i}\left(\sigma_{1}, \sigma_{2}\right), \\
X_{a^{k \gamma}}^{i}\left(\sigma_{1}, \sigma_{2}+1\right)=X_{a^{k \pi(\gamma)}}^{i}\left(\sigma_{1}+s(k, \gamma), \sigma_{2}\right) .
\end{gathered}
$$

Then, we consider a cycle of the permutation $Q$, which must be a subset of the $r_{k}$ k-cycles of $P$. Say this cycle is of length $r$ where $1 \leq r \leq r_{k}$. Then we fuse $r$ of the above fields together to get the single field which has completely periodic boundary conditions

$$
\begin{array}{r}
X_{a^{k, r}}^{i}\left(\sigma_{1}+k, \sigma_{2}\right)=X_{a^{k, r}}^{i}\left(\sigma_{1}, \sigma_{2}\right), \\
X_{a^{k, r}}^{i}\left(\sigma_{1}, \sigma_{2}+r\right)=X_{a^{k, r}}^{i}\left(\sigma_{1}+s, \sigma_{2}\right) .
\end{array}
$$

where $s=\sum_{\gamma} s(k, \gamma) \bmod k$ is the accumulated shift for the $r$ elements in the cycle of $Q$.

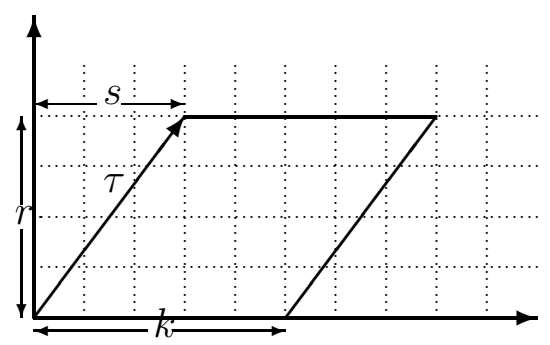

Figure 1. Connected covering torus with $r=4$, $k=5$ and $s=3$.

The space on which coordinates which are arguments of the field in eqn.(59) take values is the torus depicted in fig. 1. The contribution to the path integral of this set of fields is denoted by $Z(\tau, \bar{\tau})$ where $\tau=(s+i \nu r) / k$. It is the partition function of a super-conformal field theory living on the torus depicted in fig. 1 and having target space $R^{8}$. The boundary conditions for the Bose fields are

$$
\begin{array}{r}
X^{i}\left(\sigma_{1}+k, \sigma_{2}\right)=X^{i}\left(\sigma_{1}, \sigma_{2}\right) \\
X^{i}\left(\sigma_{1}, \sigma_{2}+r\right)=X^{i}\left(\sigma_{1}+s, \sigma_{2}\right)
\end{array}
$$

and for the Fermi fields are

$$
\begin{array}{r}
\psi\left(\sigma_{1}+k, \sigma_{2}\right)=\psi\left(\sigma_{1}, \sigma_{2}\right) \\
\psi\left(\sigma_{1}, \sigma_{2}+r\right)=(-1)^{r} \psi\left(\sigma_{1}+s, \sigma_{2}\right)
\end{array}
$$

Note that the fermions are now not all antiperiodic. When we move upward by one of the small blocks in fig. 1 they must be antiperiodic, thus the above periodicity when we move up by $r$ blocks.

It is possible to change the coordinates so that the integration region is the square torus $\left(\sigma_{1}, \sigma_{2}\right) \in([0,1),[0,1))$. The necessary coordinate transformation is

$\sigma_{1}^{\prime}=\frac{\sigma_{1}}{k}-\frac{s \sigma_{2}}{k r}, \quad \sigma_{2}^{\prime}=\frac{\sigma_{2}}{r}$

Then the metric is

$g_{a b}=\left(\begin{array}{cc}1 & \tau_{1} \\ \tau_{1} & |\tau|^{2}\end{array}\right) \quad, \quad \tau=\frac{s+i \nu r}{k}$

and the boundary conditions are

$$
\begin{array}{r}
X^{i}\left(\sigma_{1}+1, \sigma_{2}\right)=X^{i}\left(\sigma_{1}, \sigma_{2}\right) \\
X^{i}\left(\sigma_{1}, \sigma_{2}+1\right)=X^{i}\left(\sigma_{1}, \sigma_{2}\right) \\
\psi\left(\sigma_{1}+1, \sigma_{2}\right)=\psi\left(\sigma_{1}, \sigma_{2}\right) \\
\psi\left(\sigma_{1}, \sigma_{2}+1\right)=(-1)^{r} \psi\left(\sigma_{1}, \sigma_{2}\right)
\end{array}
$$

Note that the boundary condition for the Fermi field still depends on $r$. When $r$ is even, the fermion and boson have the same boundary conditions. These sectors are supersymmetric. The mode expansion of both the fermions and bosons contain zero modes. Functional integration over bosonic zero modes produces a factor of the infinite volume of $R^{8}$ and integration over the fermionic zero mode produces a factor of zero. If we were computing the Witten index, this product of infinity times zero, suitably regulated would yield the number of zero energy states of 
the supersymmetric theory. However, here, we are computing an extensive thermodynamic variable - the free energy - from which we must extract a factor of the volume of the space in order to obtain the free energy density. In this case, sectors which contain fermion zero modes do not contribute. On the other hand, when $r$ is odd, the fermions have anti-periodic boundary conditions - supersymmetry is broken by this boundary condition - and there are no fermion zero modes in the mode expansion. These sectors will survive and contribute to the partition function. Thus,

$Z(\tau, \bar{\tau})=0$ when $r$ is even

When the partition function is re-written as a sum over decompositions into cycles of the commuting permutations,

$$
\begin{array}{r}
Z=\sum_{N=0}^{\infty} \frac{1}{N !} e^{-N \beta / \sqrt{2} R} \sum_{\substack{P, Q \\
P Q=Q P}} . \\
\cdot \sum_{\text {decomp. cycles }} \prod_{(\tau, \bar{\tau}),}
\end{array}
$$

The sum over decompositions into cycles exponentiates to the exponential of a sum over connected parts. This was shown explicitly in [1] and also happens for a related expression in ref. [10]. In the end, the free energy of the matrix string theory is given by the expression

$$
F=-\frac{1}{\beta} \sum_{N=0}^{\infty} \sum_{\substack{k r=N \\ r \text { odd }}} \sum_{s=0}^{N / r-1} \frac{e^{-\frac{N \beta}{\sqrt{2} R}}}{N} Z(\tau, \bar{\tau})
$$

Note that $r$ gives the height of the torus, in the $\sigma_{2}$ direction and, taking into account eqn. (65) and the discussion before it, $r$ must be odd.

With appropriate re-labeling of the integers, and evaluating the functional integral $Z(\tau, \bar{\tau})$, the expression (67) is identical to 29), the expression for the free energy of the DLCQ type II superstring. Note that, in comparing this with eqn.(29), rather than $1 / k^{2}$ appearing in the summand in eqn.(67), there is $1 / N=1 / k r$ where we label $k=N / r$. This difference of a factor of $k / r$ is absorbed in a factor of $1 / \tau_{2}$ in eqn. 29.

\section{Remarks}

Given the good agreement of the genus 1 string partition function with the Dijkgraaf-VerlindeVerlinde limit of the matrix string model, it would be interesting to check it a higher genus. A concrete question which could be asked is whether the two-point correlator of the twist operator which ref.[23] suggested was responsible for interactions in the matrix string model could reproduce the genus 2 type II string partition function with (12) inserted. In fact, it has been found that, in the large $N$ limit, the correlation function of twist operators on a symmetric orbifold indeed correctly reproduces four-string amplitudes [39]41.

In addition, other tests of the matrix model are possible. In ref. [t the analysis of the partition function was carried out in a constant background B-field. This is a solvable deformation of closed string theory on Minkowski space. It was found that a constraint similar to (12) appeared in the string path integral. Also, the modification of the matrix string model due to the presence of a constant $B$ field was found and the zero string coupling limit of that model was again shown to have identical thermodynamic partition function to the type II string theory. It would be interesting to test this idea with other solvable deformations of string theory. Some good examples are string theories with D-branes and electromagnetic fields (for a review see ref. [42]) where, some interesting behavior is observed in the finite temperature case [43].

It is well known that string theories, because of their exponentially increasing density of states, have a limiting upper temperature. Interacting strings are thought to undergo a phase transition at that temperature 15$]$ 44 45 . It was shown in ref.11 that the Hagedorn temperature is the same in the DLCQ as it is in ordinary light-cone or covariant quantization of the string. It comes from a divergence in the sum over $N$ in equations like eqn.(42) or eqn.(26). It was found in ref.[5] that when a constant external $B$-field is switched on, the Hagedorn temperature also depends on $B$ in a way which was puzzling - the processes of decompactifying the light-cone and switching on $B$ 
did not commute with each other. An explanation which was given there was the non-extensive behavior of the Hagedorn transition.

Another interesting question to do with the Hagedorn transition is to ask where it would come from in the matrix model. The matrix model had partition function

$Z[\beta]=\sum_{N=0}^{\infty} e^{-\frac{\beta N}{\sqrt{2} R}} \int[d A d X d \psi] e^{-S_{E}\left[A, X^{i}, \psi\right]}$

and again, one expects that the only possible source of Hagedorn behavior is in the potential divergence of the summation over $N$. Since the partition function in the above expression is a sum over Yang-Mills partition functions the only possible source of divergence from the sum over $N$ is governed by the large $N$ limit of the Yang-Mills partition function.

Typically, this is bad news. In the generic large $\mathrm{N}$ limit, the Yang-Mills theory free energy goes like

$$
\beta F \sim-N^{2} \cdot \text { numbers }
$$

simply because there are $N^{2}$ degrees of freedom. This means that the partition sum typically diverges! One needs some very special tuning of parameters (such as the Dijkgraaf-Verlinde-Verlinde limit of $\left.g_{s} \rightarrow 0\right)$ if it is to converge. Even in the limit which gets free matrix strings, it goes like

$$
\beta F_{\mathrm{DVV}} \sim-N \beta_{H} / R
$$

where $\beta_{H}$ is the inverse Hagedorn temperature and the partition sum converges only if the temperature is low enough $\left(\beta>\beta_{H}\right.$.

To what do we attribute the generic divergence? One suspect might be the nucleation of black holes which would destroy the thermal ensemble. Estimates of the $N$-dependence of black hole free energy in matrix theory [46]- 49 show that it typically grows faster than $N$ (and must be negative).

Finally, we note that, in ref.12 they found a phase transition in a discrete gauge theory. They interpreted the gauge theory as a statistical model of branched covers of a triangulated Riemann surface on one hand and a random walk on the gauge group on the other hand. It is easy to see that that phase transition coincides with the Hagedorn phase transition of our DLCQ thermal partition functions if we interpret them as statistical models of branched covers. (The basic difference between this models and those of ref.[12] is that the string models have continuous rather than triangulated Riemann surfaces, so there are continuous moduli to be integrated over to get the partition function).

\section{REFERENCES}

1. G. Grignani and G. W. Semenoff, "Thermodynamic partition function of matrix superstrings," Nucl. Phys. B 561, 243 (1999) [arXiv:hep-th/9903246].

2. G. Grignani, P. Orland, L. D. Paniak and G. W. Semenoff, "Matrix theory interpretation of DLCQ string worldsheets," Phys. Rev. Lett. 85, 3343 (2000) arXiv:hep-th/0004194.

3. G. W. Semenoff, "World-sheet of the discrete light front string," arXiv:hep-th/0009011.

4. G. Grignani, M. Orselli and G. W. Semenoff, "Matrix strings in a B-field," JHEP 0107, 004 (2001) arXiv:hep-th/0104112.

5. G. Grignani, M. Orselli and G. W. Semenoff, "The target space dependence of the Hagedorn temperature," arXiv:hep-th/0110152.

6. I. K. Kostov, M. Staudacher and T. Wynter, "Complex matrix models and statistics of branched coverings of 2D surfaces," Commun. Math. Phys. 191, 283 (1998) arXiv:hepth/9703189.

7. M. Billo, M. Caselle, A. D'Adda and P. Provero, "Matrix string states in pure 2D Yang-Mills theories," Nucl. Phys. B 543, 141 (1999) arXiv:hep-th/9809095.

8. M. Billo, M. Caselle, A. D'Adda and P. Provero, "2D Yang-Mills theory as a matrix string theory," arXiv:hep-th/9901053.

9. M. Billo, A. D'Adda and P. Provero, "Matrix strings from generalized Yang-Mills theory on arbitrary Riemann surfaces," Nucl. Phys. B 576, 241 (2000) arXiv:hep-th/9911249.

10. M. Billo, M. Caselle, A. D'adda and P. Provero, "Generalized two-dimensional Yang-Mills theory is a matrix string theory," Nucl. Phys. Proc. Suppl. 88, 142 (2000) 
arXiv:hep-th/0001076.

11. M. Billo, A. D'Adda and P. Provero, "Branched coverings and interacting matrix strings in two dimensions," Nucl. Phys. B 616, 495 (2001) [arXiv:hep-th/0103242].

12. A. D'Adda and P. Provero, "Two-dimensional gauge theories of the symmetric group $\mathrm{S}(\mathrm{n})$ in the large-n limit," arXiv:hep-th/0110243.

13. N. Seiberg, "Why is the matrix model correct?," Phys. Rev. Lett. 79, 3577 (1997) arXiv:hep-th/9710009.

14. A. Sen, "D0 branes on $T(n)$ and matrix theory," Adv. Theor. Math. Phys. 2 (1998) 51 arXiv:hep-th/9709220.

15. J. J. Atick and E. Witten, "The Hagedorn Transition And The Number Of Degrees Of Freedom Of String Theory," Nucl. Phys. B 310, 291 (1988).

16. A. Bilal, "A comment on compactification of M-theory on an (almost) light-like circle," Nucl. Phys. B 521, 202 (1998) arXiv:hepth/9801047].

17. A. Bilal, "DLCQ of M-theory as the lightlike limit," Phys. Lett. B 435, 312 (1998) arXiv:hep-th/9805070.

18. J. Polchinski, "Evaluation Of The One Loop String Path Integral," Commun. Math. Phys. 104, 37 (1986).

19. J.-P. Serre, A Course in Arithmetic, SpringerVerlag, New York, 1973.

20. R. Dijkgraaf, G. W. Moore, E. Verlinde and H. Verlinde, "Elliptic genera of symmetric products and second quantized strings," Commun. Math. Phys. 185, 197 (1997) arXiv:hep-th/9608096.

21. R. Dijkgraaf, "Fields, strings, matrices and symmetric products," arXiv:hep-th/9912104.

22. H. Fuji and Y. Matsuo, "Open string on symmetric product," Int. J. Mod. Phys. A 16, 557 (2001) arXiv:hep-th/0005111.

23. R. Dijkgraaf, E. Verlinde and H. Verlinde, "Matrix string theory," Nucl. Phys. B 500, 43 (1997) arXiv:hep-th/9703030.

24. W. Taylor, "M(atrix) theory: Matrix quantum mechanics as a fundamental theory," Rev. Mod. Phys. 73, 419 (2001) arXiv:hepth/0101126.

25. W. I. Taylor, "The M(atrix) model of M- theory," arXiv:hep-th/0002016.

26. T. Banks, W. Fischler, S. H. Shenker and L. Susskind, "M theory as a matrix model: A conjecture," Phys. Rev. D 55, 5112 (1997) arXiv:hep-th/9610043.

27. L. Susskind, "Another conjecture about M(atrix) theory," arXiv:hep-th/9704080.

28. J. Ambjorn, Y. M. Makeenko and G. W. Semenoff, "Thermodynamics of D0-branes in matrix theory," Phys. Lett. B 445, 307 (1999) arXiv:hep-th/9810170.

29. T. Wynter, "Gauge fields and interactions in matrix string theory," Phys. Lett. B 415, 349 (1997) arXiv:hep-th/9709029.

30. S. B. Giddings, F. Hacquebord and H. Verlinde, "High energy scattering and D-pair creation in matrix string theory," Nucl. Phys. B 537, 260 (1999) arXiv:hep-th/9804121.

31. T. Wynter, "Anomalies and large $\mathrm{N}$ limits in matrix string theory," Phys. Lett. B 439, 37 (1998) arXiv:hep-th/9806173.

32. P. Brax and T. Wynter, "Limits of matrix theory in curved space," Nucl. Phys. B 546, 182 (1999) arXiv:hep-th/9806176].

33. G. Bonelli, L. Bonora and F. Nesti, "String interactions from matrix string theory," Nucl. Phys. B538, 100 (1999) hep-th/9807232.

34. I. K. Kostov and P. Vanhove, "Matrix string partition functions," Phys. Lett. B444, 196 (1998) hep-th/9809130].

35. G. Bonelli, L. Bonora, F. Nesti and A. Tomasiello, "Matrix string theory and its moduli space," Nucl. Phys. B554, 103 (1999) hep-th/9901093.

36. T. Wynter, "High energy scattering amplitudes in matrix string theory," Nucl. Phys. B 580, 147 (2000) arXiv:hep-th/9905087.

37. P. Brax, "The supermoduli space of matrix string theory," JHEP 0007, 020 (2000) arXiv:hep-th/9912103.

38. R. Schiappa, "Matrix strings in weakly curved background fields," Nucl. Phys. B 608, 3 (2001) arXiv:hep-th/0005145.

39. G. Arutyunov, S. Frolov and A. Polishchuk, "On Lorentz invariance and supersymmetry of four particle scattering amplitudes in $\mathrm{S}(\mathrm{N})$ $\mathrm{R}^{* * 8}$ orbifold sigma model," Phys. Rev. D 60, 066003 (1999) arXiv:hep-th/9812119]. 
40. G. E. Arutyunov and S. A. Frolov, "Four graviton scattering amplitude from $\mathrm{S}(\mathrm{N})$ $\mathrm{R}^{* *} 8$ supersymmetric orbifold sigma model," Nucl. Phys. B 524, 159 (1998) arXiv:hepth/9712061.

41. G. E. Arutyunov and S. A. Frolov, "Virasoro amplitude from the $\mathrm{S}(\mathrm{N}) \mathrm{R}^{* *} 24$ orbifold sigma model," Theor. Math. Phys. 114, 43 (1998) arXiv:hep-th/9708129].

42. J. Ambjorn, Y. M. Makeenko, G. W. Semenoff and R. J. Szabo, "String theory in electromagnetic fields," arXiv:hepth/0012092.

43. J. Ambjorn, Y. Makeenko, G. W. Semenoff and R. J. Szabo, "Screening and D-brane dynamics in finite temperature superstring theory," Phys. Rev. D 60, 106009 (1999) arXiv:hep-th/9906134.

44. B. Sathiapalan, "Vortices On The String World Sheet And Constraints On Toral Compactification," Phys. Rev. D 35, 3277 (1987).

45. Y. I. Kogan, "Vortices On The World Sheet And String's Critical Dynamics," JETP Lett. 45, 709 (1987) [Pisma Zh. Eksp. Teor. Fiz. 45, 556 (1987)].

46. T. Banks, W. Fischler, I. R. Klebanov and L. Susskind, "Schwarzschild black holes from matrix theory," Phys. Rev. Lett. 80, 226 (1998) arXiv:hep-th/9709091.

47. I. R. Klebanov and L. Susskind, "Schwarzschild black holes in various dimensions from matrix theory," Phys. Lett. B 416, 62 (1998) arXiv:hep-th/9709108.

48. M. Li, "Matrix Schwarzschild black holes in large N limit," JHEP 9801, 009 (1998) arXiv:hep-th/9710226.

49. T. Banks, W. Fischler, I. R. Klebanov and L. Susskind, "Schwarzschild black holes in matrix theory. II," JHEP 9801, 008 (1998) arXiv:hep-th/9711005. 